\title{
Searching for Black Holes with the ATLAS Detector
}

\author{
James Frost \\ On behalf of the ATLAS Collaboration \\ University of Cambridge, Cavendish Laboratory, Madingley Road, Cambridge, CB3 OHE, UK.
}

\begin{abstract}
Models with extra spacial dimensions offer a new way to address problems in the Standard Model. The most spectacular manifestation of these would be the production and decay of microscopic black holes. The outlook and potential of the ATLAS detector at the LHC to discover and measure black holes in models with large extra dimensions is presented.
\end{abstract}

Keywords: ATLAS, LHC, Extra Dimensions, Black Holes

PACS: $04.70 .-\mathrm{s}, 12.60 . \mathrm{i}, 14.80 .-\mathrm{j}$

\section{INTRODUCTION}

Arkani-Hamed, Dimopoulos and Dvali (ADD) and Randall and Sundrum (RS) pioneered the solution of the hierarchy problem by using extra-dimensional space, whose geometry generates the hierarchy ${ }^{1}$. The apparent weakness of gravity results from its ability to propagate into the bulk; Standard Model (SM) fields are confined to our 3-brane. For large extra (ADD) or strongly warped (RS) extra dimensions, the scale of gravitational interactions can be as low as the $\mathrm{TeV}$-scale, opening the intriguing possibility of producing black holes at the LHC. Their discovery and investigation would test general relativity and extra-dimensions, and even probe quantum gravity.

Especially for a low number of extra-dimensions $(n)$, the minimum Planck scale in ADD models is highly constrained. Constraints on these models come from a variety of sources, from the Tevatron data, tabletop experiments and astrophysical measurements from supernovae and neutron star cooling, cosmic and gamma-ray data.

A general formulation of black hole $(\mathrm{BH})$ production is extremely complex. Semi-classical assumptions, valid only above the Planck scale, are necessary to enable a quantitative description and predictions. Consequently, a minimum BH mass must be imposed in our simulation, above which these conditions are satisfied. This study used the CHARYBDIS black-hole event generator [2], which uses full spin-dependent grey body factors to calculate the energy spectrum of the emitted particles. It models only the Schwarzschild phase, during which a non-rotating $\mathrm{BH}$ emits particles by Hawking radiation, losing mass and increasing its Hawking temperature. Graviton emission and black hole angular momentum are not modelled. Generator switches allow some of the theoretical uncertainties to be investigated.

\footnotetext{
${ }^{1}$ Please see [1] for further details and comprehensive references.
}

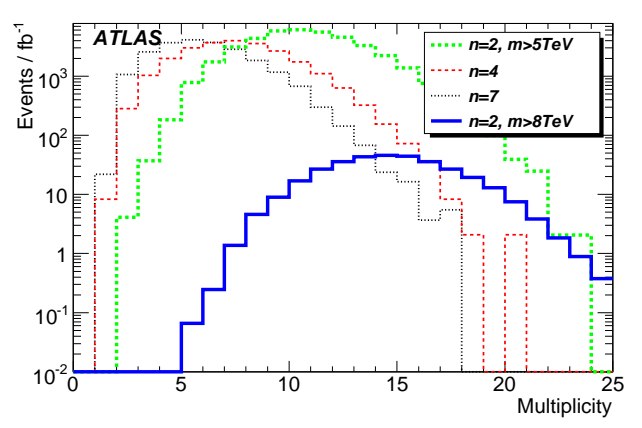

FIGURE 1. Particle Multiplicities in BH datasets, with $n$ extra dimensions and minimum BH mass $m$.

\section{RESULTS AND DISCUSSION}

Due to the high Hawking temperature and mass scale, semi-classical black holes tend to emit particles with very high energy and transverse momentum $\left(P_{T}\right)$. Given a minimum black hole mass, the behaviour is driven primarily by the Hawking temperature: for smaller $n$, the greater Hawking temperature results in a smaller number of more energetic particles being produced, for a black hole of given input mass. This has a powerful effect on the multiplicity, transverse momentum and event shape distributions. For greater experimental and methodological detail, the interested reader is referred to [1].

Though black holes are predicted to produce all SM particles according to their degrees of freedom, at the LHC such a "democratic decay" is only loosely achieved, due to the usually charged and coloured input state. A high- $P_{T}$ jet trigger is highly efficient $(>99 \%)$ for $\mathrm{BH}$ events, due to the presence of a hard jet in virtually every event. Event shape variables have been heralded as a useful method of distinguishing BH events from SM background, due to the multiple emissions and isotropic angular distributions in $\mathrm{BH}$ events. We find the discriminat- 


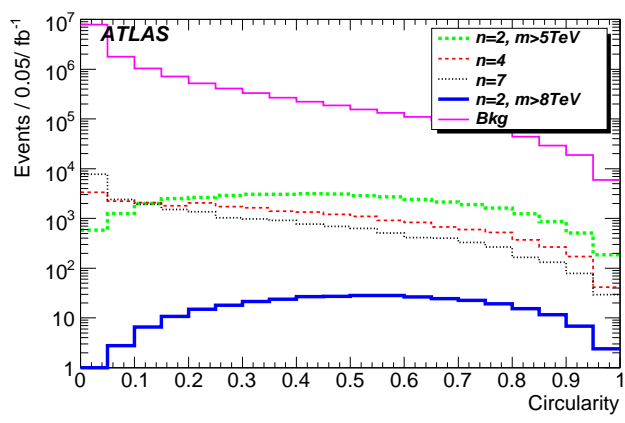

FIGURE 2. Circularity in BH and SM background events

ing power of such variables to be lessened by two factors. Firstly, the large background cross-sections cause the tail of their distributions to overlap with much of the black hole distributions, and secondly different black hole samples can have widely varying multiplicities and event shape distributions (Figures 1 and 2). We find that this sensitivity to theoretical uncertainties and model parameters, coupled with the overlap of the signal and background distributions, makes them ill-suited as hard cut variables, although some discriminatory power remains.

The ATLAS detector has sophisticated methods of particle identification, of which we make full use. Two highly efficient methods of signal selection were determined, which are complementary. Both demonstrate high signal efficiency and good background rejection for all black hole samples and SM backgrounds. An additional cut requiring a high $P_{T}$ lepton (e or $\mu$ ) suppresses the background further. The first relies on the high mass scale of black hole events, and is based on a cut on the scalar summation of the $P_{T}$ of all objects, requiring $\sum\left|P_{T}\right|>2.5 \mathrm{TeV}$ and the presence of a lepton with $P_{T}>50 \mathrm{GeV}$. This results in the efficiencies and rejections shown Table 1. Errors shown on the signal samples are statistical only, the theoretical uncertainties on their cross-sections are large. The second uses the high- $P_{T}$ object multiplicity, requiring at least four with a $P_{T}$ above $200 \mathrm{GeV}$; a second cut requires one to be a lepton. This latter method is complementary and provides similar performance and potential, having a different sensitivity to experimental and theoretical uncertainties. For reasons of brevity and consistency, a duplicate discovery plot is omitted - please refer to [1] for further details. We reconstruct the black hole mass from the four-vectors of all final state reconstructed particles and missing $E_{T}$.

Our simulation will underestimate the amount of missing $E_{T}$ produced in black hole events, since graviton emission is ignored. Nonetheless BH samples produce a large range of missing $E_{T}$, with tails extending beyond $2 \mathrm{TeV}$. This feature, coupled with the large cross-section, is uncharacteristic of other new physics scenarios, and

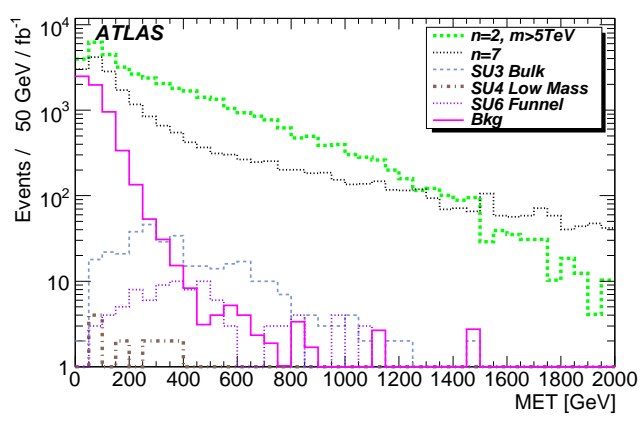

FIGURE 3. Missing $E_{T}$ after a cut $\Sigma\left|P_{T}\right|>2.5 \mathrm{TeV}$

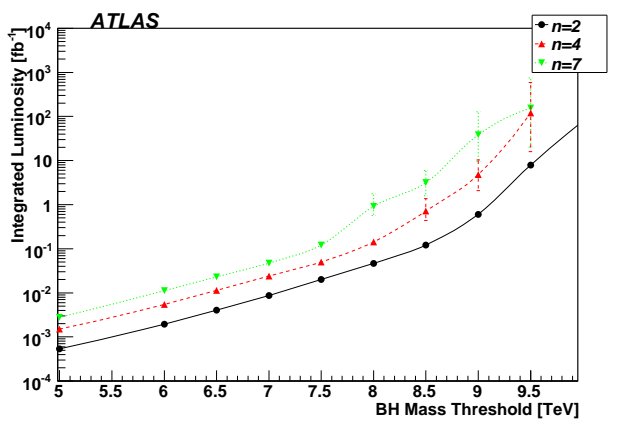

FIGURE 4. Discovery Reach for semi-classical black holes

potentially distinguishes BH models from SUSY ones. Figure 3 shows missing $E_{T}$ distributions after a requirement of $\sum\left|P_{T}\right|>2.5 \mathrm{TeV}$ for black hole samples, SM background and three common SUSY models. Though little SUSY or SM background exists above a missing $E_{T}$ of $600 \mathrm{GeV}$, the robustness of such a selection is limited: there are many uncertainties and assumptions in our modelling that will affect the missing $E_{T}$ distribution, and most importantly, accurate $\mathrm{BH}$ mass reconstruction is aided by limiting missing $E_{T}$ to be less than $100 \mathrm{GeV}$, thereby achieving mass resolutions of $\approx 320 \mathrm{GeV}$.

Since our model requires us to make semi-classical assumptions, valid only above the Planck scale, producing a robust discovery potential is difficult, for this lack of theoretical understanding prohibits us from modelling the threshold, Transplanckian region. To account for this, we impose a threshold cut on the true BH mass, and do not attempt to account for any additional signal from $\mathrm{BH}$ masses below this. A conservative estimate of the discovery reach can be calculated by varying this threshold cut, keeping selection cuts constant (Figure 4).

Should a discovery be made, we will wish to place constraints upon the model realised in nature. A method with the potential to constrain $n$ was described in [3], provided that a measurement of the Planck scale (from the inclusive cross-section or otherwise) has been made. 
TABLE 1. Acceptance for signal and background datasets

\begin{tabular}{ccccc}
\hline dataset & $\begin{array}{c}\text { events before } \\
\text { cuts }\left(1 \mathrm{fb}^{-1}\right)\end{array}$ & $\begin{array}{c}\text { events passing } \sum\left|P_{T}\right| \\
>2.5 \mathrm{TeV}\left(1 \mathrm{fb}^{-1}\right)\end{array}$ & $\begin{array}{c}\text { after requiring lepton } \\
\text { events in }\left(1 \mathrm{fb}^{-1}\right)\end{array}$ & acceptance \\
\hline $\mathrm{n}=2, \mathrm{~m}>5 \mathrm{TeV}$ & $40690 \pm 116$ & $39182 \pm 305$ & $18618 \pm 146$ & 0.46 \\
$\mathrm{n}=4, \mathrm{~m}>5 \mathrm{TeV}$ & $24320 \pm 53$ & $22591 \pm 222$ & $6668 \pm 83$ & 0.27 \\
$\mathrm{n}=7, \mathrm{~m}>5 \mathrm{TeV}$ & $22270 \pm 42$ & $20082 \pm 195$ & $3574 \pm 60$ & 0.17 \\
$\mathrm{n}=2, \mathrm{~m}>8 \mathrm{TeV}$ & $338.2 \pm 1$ & $338.1 \pm 2.5$ & $212 \pm 16$ & 0.63 \\
$t \bar{t}$ & $833000 \pm 100000$ & $23.6_{-6.7}^{+12.2}$ & $8.2_{-2.43}^{+2.43}$ & $9.8 \times 10^{-6}$ \\
$\mathrm{QCD}$ dijets & $12836131 \pm 3740000$ & $5899_{-1771}^{+1737}$ & $5.37_{-2.02}^{+3.25}$ & $4.3 \times 10^{-7}$ \\
$\mathrm{~W}(\rightarrow \mathrm{e} v, \mu v)+\geq 2$ jets & $1911300 \pm 38200$ & $12.3_{-1.8}^{+9.0}$ & $4.67_{-0.93}^{+8.75}$ & $2.4 \times 10^{-6}$ \\
$\mathrm{Z}(\rightarrow \mathrm{ee}, \mu \mu)+\geq 3$ jets & $51807 \pm 1036$ & $2.75_{-2.01}^{+2.02}$ & $2.57_{-0.64}^{+0.95}$ & $5.0 \times 10^{-5}$ \\
\hline
\end{tabular}

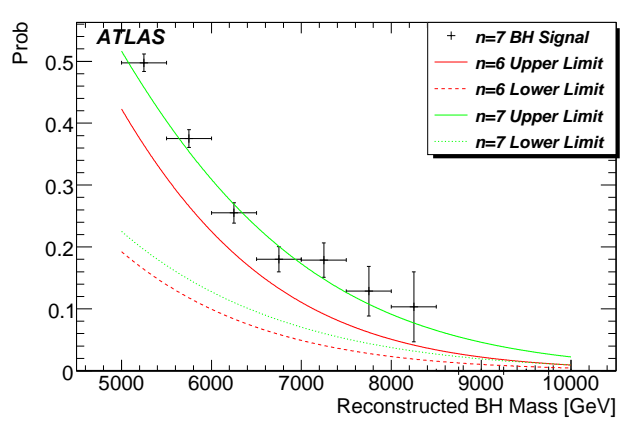

FIGURE 5. Constraining the number of extra dimensions

It is here made compatible with background rejection cuts. The standard cuts described earlier are unsuitable for this, for they bias the sample in favour of a larger number of emissions; requiring $\Sigma\left|P_{T}\right|>3.5 \mathrm{TeV}$ is effective and unbiased. Accurate mass resolution is necessary, so the missing $E_{T}$ cut is applied, despite its effect on signal efficiency. The data are consistent with expectation, but greater luminosity than the $1 \mathrm{fb}^{-1}$ shown in Figure 5 will be necessary for a conclusive result.

\section{Next Steps}

Recent theoretical progress has begun to produce more sophisticated tools and Monte-Carlo generators [4][5]. The calculation of grey-body factors for rotating BHs [6] allows their particle emission to be modelled, which can have large effects on particle distributions - exemplified by Figure 6. In addition, there has been progress in the modelling of their production and decay.

\section{CONCLUSIONS}

The ATLAS detector at the LHC provides an unprecedented opportunity to investigate the presence of extra spatial dimensions and microscopic black holes. Meth-

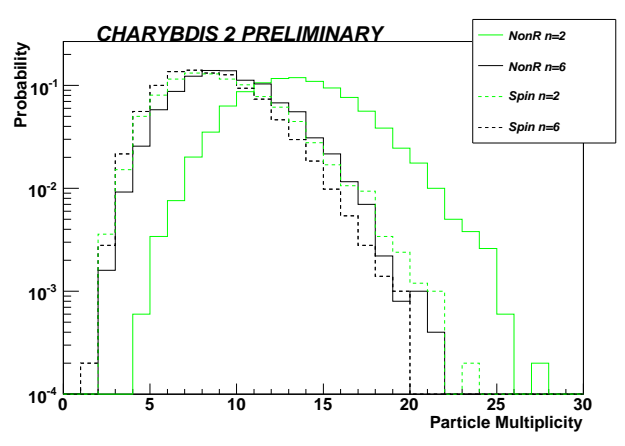

FIGURE 6. Particle multiplicity distributions for spinning and non-rotating $\mathrm{BH}$

ods through which a black hole signal can be separated from SM backgrounds and the black hole mass reconstructed have been determined; techniques by which constraints may be put upon the number of extra dimensions have been made consistent with them. Subject to the validity of the semi-classical cross-section estimates, black holes above a $5 \mathrm{TeV}$ production threshold can be discovered with a few $\mathrm{pb}^{-1}$ of data, whereas $1 \mathrm{fb}^{-1}$ would be sufficient were the threshold to be $8 \mathrm{TeV}$. The difficulty in making predictions near the Planck scale remains the major obstacle to a more precise understanding.

\section{REFERENCES}

1. The ATLAS Collaboration, CERN-OPEN-2008-020, Geneva, 2008, to appear.

2. C. M. Harris, P. Richardson and B. R. Webber, J. High Energy Phys. 0308 (2003) 033;

3. C. M. Harris, M. J. Palmer, M. A. Parker, P. Richardson, A. Sabetfakhri and B. R. Webber, J. High Energy Phys. 0505 (2005) 053;

4. De-Chang Dai, Glenn Starkman, Dejan Stojkovic, Cigdem Issever, Eram Rizvi and Jeff Tseng, Phys. Rev. D 77 076007 (2008)

5. M. Casals, S. R. Dolan, J. Frost, J. R. Gaunt, M. A. Parker, M. O. P. Sampaio, B. R. Webber, (in preparation)

6. D. Ida, K.-Y. Oda and S. C. Park, P. R. D. 73 (2006) 124022 